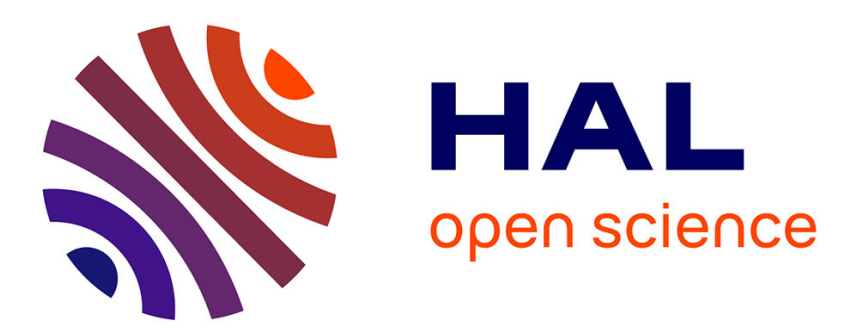

\title{
Nonlinear modal analysis of mistuned periodic structures subjected to dry friction
}

Colas Joannin, Benjamin Chouvion, Fabrice Thouverez, Moustapha Mbaye, Jean-Philippe Ousty

\section{- To cite this version:}

Colas Joannin, Benjamin Chouvion, Fabrice Thouverez, Moustapha Mbaye, Jean-Philippe Ousty. Nonlinear modal analysis of mistuned periodic structures subjected to dry friction. Journal of Engineering for Gas Turbines and Power, 2015, 10.1115/1.4031886 . hal-01260983

\section{HAL Id: hal-01260983 \\ https://hal.science/hal-01260983}

Submitted on 22 Jan 2016

HAL is a multi-disciplinary open access archive for the deposit and dissemination of scientific research documents, whether they are published or not. The documents may come from teaching and research institutions in France or abroad, or from public or private research centers.
L'archive ouverte pluridisciplinaire HAL, est destinée au dépôt et à la diffusion de documents scientifiques de niveau recherche, publiés ou non, émanant des établissements d'enseignement et de recherche français ou étrangers, des laboratoires publics ou privés. 


\title{
Nonlinear modal analysis of mistuned periodic structures subjected to dry friction
}

\author{
Colas Joannin $^{\mathrm{a}, \mathrm{b}, *}$, Benjamin Chouvion ${ }^{\mathrm{a}}$, Fabrice Thouverez ${ }^{\mathrm{a}}$, Moustapha Mbaye $^{\mathrm{b}}$, Jean-Philippe Ousty ${ }^{\mathrm{b}}$ \\ ${ }^{a}$ École Centrale de Lyon, LTDS, CNRS UMR 5513, 69130 Écully, France \\ ${ }^{b}$ Turbomeca, Safran Group, 64511 Bordes, France
}

\begin{abstract}
This paper deals with the dynamics of a cyclic system, representative of a bladed-disk subjected to dry friction forces, and exhibiting structural mistuning. The nonlinear complex modes are computed by solving the eigenproblem associated to the free response of the whole structure, and are then used to better understand the forced response to a traveling wave excitation. Similarly to the underlying linear system, the tuned model possesses pairs of modes that can be linearly combined to form traveling waves, unlike those of the mistuned structure. However, due to the nonlinearity, the modal properties are not constant but vary with the vibration amplitude in both cases. A qualitative analysis is also performed to assess the impact of the mistuning magnitude on the response, and suggests that further statistical investigations could be of great interest for the design of bladed disks, in terms of vibration mitigation and robustness.
\end{abstract}

Keywords: nonlinear modes, mistuning, friction, bladed disk

\section{Introduction}

Bladed disks are key components of turbomachinery. They achieve the momentum transfer between the gas flow going through a turbomachine and the rotating shafts on which are mounted axial turbines and compressors. In operating conditions, these components undergo many sources of excitation, making of vibration mitigation a challenge of great interest in bladed disk design. In this context, contact phenomena have proved efficient in coping with vibrations, and their strongly nonlinear nature is now better understood thanks to numerous numerical [1-4] and experimental [5-9] investigations.

Even though the manufacturing of blades must comply with stringent tolerancing in order to ensure an optimum efficiency, the process inevitably results in slight material discrepancies which break the cyclic symmetry of the "ideal" structure, and may lead to a complex behavior with a potential energy localization on a few blades. This phenomenon is commonly referred to as mistuning, and has been thoroughly studied over the years for linear systems, in terms of phenomenology, reduced order modeling, identification, and robustness analysis[10-14].

Linear modal analysis is nowaday well established in both the academic and industrial fields, providing researchers and engineers with a multitude of theoretical, numerical, and experimental tools to understand and predict the dynamic behavior of linear systems [15]. During the last decades, a lot of effort has been put into building a nonlinear analog for conservative structures. Since Rosenberg [16] addressed this branch of nonlinear dynamics, many contributions have led to a rich and topical framework thoroughly reviewed by Kershen et al. in [17], including Shaw and Pierre's [18] effort to extend Rosenberg's definition of a nonlinear mode to damped systems. The work achieved by Vakakis et al. [19-21] on the dynamics of cyclic systems with nonlinear stiffnesses showed how interesting nonlinear normal modes are in the study of periodic structures, revealing that localization phenomena can occur even in a perfectly tuned assembly. However, the type of nonlinearities dealt with in these papers differ from dry friction in that they are conservative.

\footnotetext{
${ }^{*}$ Corresponding author

Email address: colas.joannin@doctorant.ec-lyon.fr (Colas Joannin)
} 
In order to compute nonlinear modes in the presence of dissipative nonlinearities, such as dry friction, a new approach was developed by Laxalde and Thouverez [22] and applied to the finite element model of a compressor blade with contact interfaces at the root. Krack et al. [23] have implemented a similar method to perform a nonlinear modal synthesis on a bladed disk with shroud contact, enforcing cyclic boundary conditions so that the computation only needed to be carried out on a single sector. The use of nonlinear complex modes has proven very interesting in both cases and applicable to large-scale models, making of nonlinear modal analysis of such structures an actual perspective for the industry.

In the present paper, the dynamics of periodic structures undergoing dry friction nonlinearities and subjected to mistuning is investigated by means of a simplified model of bladed-disk. The behavior of such systems was already studied by Wei and Pierre in [24], by computing the forced response to a traveling wave excitation. Here, the methodology developed in [22] is used to compute the nonlinear modes besides the forced response, and thus provide information as to the intrinsic dynamics of the structure, similarly to the work achieved by Vakakis et al. for conservative nonlinearities. The impact of structural mistuning on the modal properties of the nonlinear system is addressed, and a qualitative analysis of the mistuning magnitude is carried out. As a consequence of the mistuning, the computation of the forced response and of the nonlinear complex modes is performed on the whole structure, and not on a single sector as in [23].

\section{Nonlinear complex modes}

In accordance with the definition given in [22], a nonlinear complex mode refers hereafter to a periodic or pseudo-periodic motion of an autonomous nonlinear system, such as Eqn. (1). This system of differential equations could be the governing equations of motion of a discretized structure under no external load, with $\mathbf{M}, \mathbf{C}, \mathbf{K}$ the mass, viscous damping, and linear stiffness matrices, respectively, and $\mathbf{f}_{n l}$ a term of internal nonlinear forces.

$$
\mathbf{M} \ddot{\mathbf{x}}(t)+\mathbf{C} \dot{\mathbf{x}}(t)+\mathbf{K x}(t)+\mathbf{f}_{n l}(\mathbf{x}, \dot{\mathbf{x}})=\mathbf{0}
$$

In order to account of the dissipation, and of the potential phase shift between the degrees of freedom (DOFs), the nonlinear modes are sought in the form of a decaying multi-harmonic oscillation, given by Eqn. (2).

$$
\mathbf{x}(t)=\sum_{k=0}^{\infty} \mathrm{e}^{-k \beta t}\left(\mathbf{a}_{k} \cos (k \omega t)+\mathbf{b}_{k} \sin (k \omega t)\right)
$$

The terms $\mathbf{a}_{k}$ and $\mathbf{b}_{k}$ are vectors containing the coefficients of the $\mathrm{k}$-th cosine and sine harmonics of the mode, respectively, for all DOFs. The coefficients $\beta$ and $\omega$ are referred to as modal damping and natural frequency. By analogy with linear complex modes, these two coefficients are related to an eigenvalue $\lambda$ associated to the nonlinear mode, which may be complex if dissipative phenomena are taken into account [22], as written in Eqn. (3).

$$
\lambda=-\beta+\mathrm{i} \omega
$$

Nonlinear modes differ from their linear analog in that they are energy-dependent. In other words, for a given mode, the eigenvalue $\lambda$ and corresponding free oscillation of Eqn. (2) may vary with the level of activation of the nonlinearity. At low level, the behavior of the system is close to that of the underlying linear model, justifying the use of a linear modal analysis. However, when the nonlinearity is moderately or highly activated, the actual dynamics of the structure may significantly differ from that predicted by a linear analysis, which could jeopardize the design of the whole structure, should a linear model be kept. A nonlinear modal analysis aims at superseding linear modes in such cases, for which nonlinearities should not be overlooked.

\section{Practical computation}

As a consequence of their energy-dependency, the computation of nonlinear modes requires some specific treatment, resulting in more computational effort than a linear modal analysis. This section provides an overview of the main steps of the calculation. Most of the method is inspired by the work achieved by Laxalde and Thouverez, and further details can be found in [22]. 
The nonlinear modes are obtained through the computation of the solutions of Eqn.(1) by means of the Harmonic Balance Method (HBM)[25]. The infinite series expansion of $\mathbf{x}$ in Eqn. (2) must first be truncated to a finite number $N_{h}$ of retained harmonics. Then, substituting the expression of $\mathbf{x}$ into Eqn. (1), and applying a Galerkin procedure on the projection basis $\boldsymbol{\psi}=\{\cos (k \omega t), \sin (k \omega t)\}_{\forall k \in \llbracket 0, N_{h} \rrbracket}$ by means of the following inner product

$$
\langle f, g\rangle=\frac{2}{T} \int_{0}^{T} f(t) g(t) d t
$$

with $T=2 \pi / \omega$, yields an algebraic system of equations on the variables $\hat{\mathbf{x}}, \omega$, and $\beta$ :

$$
\mathbf{Z}(\omega, \beta) \hat{\mathbf{x}}+\hat{\mathbf{f}}_{n l}(\omega, \beta, \hat{\mathbf{x}})=\mathbf{0}
$$

The term $\mathbf{Z}$ is a block-tridiagonal matrix built from the structural matrices $\mathbf{M}, \mathbf{C}, \mathbf{K}$, and referred to as dynamic stiffness matrix. The variable $\hat{\mathbf{x}}$ is a vector containing the harmonic coefficient $\mathbf{a}_{k}$ and $\mathbf{b}_{k}$, sorted as follow :

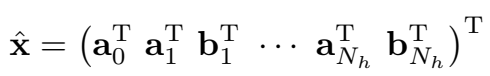

Last but not least, $\hat{\mathbf{f}}_{n l}$ is the vector of nonlinear forces in the frequency domain, the computation of which is detailed in the next paragraph. This algebraic system is solved iteratively by means of a trust-region algorithm. The analytical expression of the jacobian matrix $\mathbf{J}$ of the system is calculated beforehand in order to avoid numerical differentiations and thus improve the performance of the solver.

$$
\mathbf{J}=\left(\begin{array}{ccc}
\mathbf{Z}+\frac{\partial \hat{\mathbf{f}}_{n l}}{\partial \hat{\mathbf{x}}} & \frac{\partial \mathbf{Z}}{\partial \omega}+\frac{\partial \hat{\mathbf{f}}_{n l}}{\partial \omega} & \frac{\partial \mathbf{Z}}{\partial \beta}+\frac{\partial \hat{\mathbf{f}}_{n l}}{\partial \beta}
\end{array}\right)
$$

Practically speaking, an analytical expression of $\hat{\mathbf{f}}_{n l}$ might be cumbersome to obtain and manipulate for a frictional nonlinearity. The nonlinear term of Eqn. (5) is instead determined through an alternating frequency-time (AFT) scheme, as suggested by Cameron and Griffin [26], and performed at each iteration of the solver by means of direct (DFT) and inverse (IDFT) discrete Fourier transforms, according to the following steps :

1. IDFT on $\hat{\mathbf{x}}$ to determine $\mathbf{x}$ and $\dot{\mathbf{x}}$.

2. Computation of $\mathbf{f}_{n l}(\mathbf{x}, \dot{\mathbf{x}})$ in the time domain.

3. DFT on $\mathbf{f}_{n l}(\mathbf{x}, \dot{\mathbf{x}})$ to get $\hat{\mathbf{f}}_{n l}(\omega, \beta, \hat{\mathbf{x}})$.

It should be noticed that in order to readily perform the AFT scheme by means of DFT and IDFT matrices, the displacements $\mathbf{x}$ and velocities $\dot{\mathbf{x}}$ are assumed to be periodic when computing the nonlinear terms. In other words, the exponential term in Eqn. (2) is dropped while computing $\hat{\mathbf{x}}$, and hence the decrease in $\mathbf{x}$ and $\dot{\mathbf{x}}$ over a period of oscillation is not taken into account. This amounts to assuming that the nonlinear terms decrease at the same rate than the other variables [22].

In general, due to their energy-dependency, nonlinear modes are not hyperplanes of the configuration space. However, they can all be included in a larger family of topological objects referred to as manifolds. In other words, in a two-dimensional subspace of the configuration space, nonlinear modes may no longer be straight lines, but curves, unlike their linear analogs [16]. Nonlinear modes associated to hyperplanes of the configuration space are termed similar nonlinear modes by Rosenberg, in contrast to nonsimilar ones corresponding to curved motions in that space. In order to efficiently deal with this curvature, an arc-length continuation was used to trace the loci of the solutions [27]. This is achieved by adding the scalar equation (8) to Eqn. (5), which amounts to enforcing the solution to be on the hypersphere of the configuration space centered on the last converged solution and of radius $d s$.

$$
\|d \hat{\mathbf{x}}\|^{2}+d \omega^{2}+d \beta^{2}=d s^{2}
$$

Finally, a last equation is required to supplement Eqn. (5) and (8) and determine the unknowns $(\hat{\mathbf{x}}, \omega, \beta)$. It is possible for instance to impose the phase of the k-th harmonic of the i-th DOF, as achieved by Eqn. (9). 
This slightly differs from [22], where both the amplitude and the phase of a DOF had to be enforced since no arc-length equation supplemented Eqn. (5). The analytical expressions of the differentiations of Eqn. (8) and (9) with respect to $\hat{\mathbf{x}}, \omega$, and $\beta$ are also provided to the solver to supplement the jacobian matrix $\mathbf{J}$.

$$
\mathbf{a}_{k}(i)=\mathbf{b}_{k}(i)
$$

In order to help or simply ensure the convergence of the solver at a given step $i$ of the continuation, a suitable initialization is required. A secant predictor based on the last two computed solutions is thus used to initialize the variable $\mathbf{z}=\left(\hat{\mathbf{x}}^{\mathrm{T}}, \omega, \beta\right)^{\mathrm{T}}$, according to Eqn. (10) and Eqn. (11).

$$
\begin{aligned}
\mathbf{z}_{p} & =\mathbf{z}_{i-1}+d s \cdot \frac{\Delta \mathbf{z}}{\|\Delta \mathbf{z}\|} \\
\Delta \mathbf{z} & =\mathbf{z}_{i-1}-\mathbf{z}_{i-2}
\end{aligned}
$$

The initialization of the first step of the continuation requires then a specific treatment, since no previous solutions are available to compute the prediction $\mathbf{z}_{p}$. The nonlinear modes of interest here originating from the modes of the underlying linear system, the continuation is initialized on a linear mode $\left(\boldsymbol{\varphi}_{0}, \lambda_{0}\right)$ at low activation of the nonlinearity, by premultiplying the real and imaginary parts of the linear complex eigenvector by a small coefficient $\delta$. Insofar as it defines which nonlinear mode is to be computed, a great care should be taken in defining this starting point, especially when two linear modes have neighboring natural frequencies.

$$
\begin{aligned}
\boldsymbol{\varphi}_{0} & =\mathbf{a}_{1}+\mathrm{i} \mathbf{b}_{1} \\
\lambda_{0} & =-\beta_{0}+\mathrm{i} \omega_{0} \\
\mathbf{z}_{0} & =\left(\begin{array}{llllllll}
0 & \delta \mathbf{a}_{1}^{\mathrm{T}} & -\delta \mathbf{b}_{1}^{\mathrm{T}} & 0 & \cdots & 0 & \omega_{0} & \beta_{0}
\end{array}\right)^{\mathrm{T}}
\end{aligned}
$$

In the next section, this methodology is implemented and used to compute the nonlinear modes of a lumped-parameter model representing a simplified bladed disk undergoing dry friction nonlinearities.

\section{Phenomenological model}

In order to study the phenomenology of bladed disks subjected to structural mistuning and dry friction at moderate computational cost, a one-dimensional lumped-parameter model has been devised. It consists of several sectors such as drawn on Fig. (1), all of which made of three DOFs to account of the blade tip, middle, and root respectively, and one DOF to account of the disk. For each sector, the disk and the root undergo a frictional force, function of their relative motions, as in a blade-disk joint. Three DOFs are kept to model the blade, so as to moderate the influence of the nonlinearity on the dynamic monitored at the tip. A similar model was used in [2] to study the forced response of integrally bladed disks with friction ring dampers. A total number of six sectors is retained as a compromise between computational efficiency and representativity in terms of nodal diameters. The model is tuned so that it could account for the first family of bending modes of an actual bladed disk. The masses are defined so that the overall mass of a blade and a disk sector is approximated. The stiffnesses are then tuned to set the natural frequencies of the model. In the tuning process, a particular attention is paid to the value of the coupling stiffness between two adjacent sectors, which governs the evolution of the natural frequencies as a function of the number of nodal diameters. The value retained for each structural parameter is reported in Tab. (1). The natural frequencies of the first family of bending modes of the tuned linear system are reported in Tab. (2) for bonded and frictionless interfaces. For these tuned linear cases, $\left(f_{2}, f_{3}\right)$ and $\left(f_{4}, f_{5}\right)$ are pairs of equal natural frequencies associated to double modes with 1 and 2 nodal diameters, respectively, whereas $f_{1}$ and $f_{6}$ correspond to single modes with 0 and 3 nodal diameters, respectively.

\subsection{Excitation}

In a turbomachine, traveling wave excitations are commonly encountered, as they naturally arise from the static parts present in the gas flow. For instance, for a given rotor, the upstream stator stage generates a perturbation in the flow which may excite periodically the blades passing by. In a frame of reference fixed to the rotor, this perturbation is seen, to a first approximation, as a sine wave propagating clockwise or anticlockwise. The number of static parts from which arises the excitation defines the number of nodal diameters 


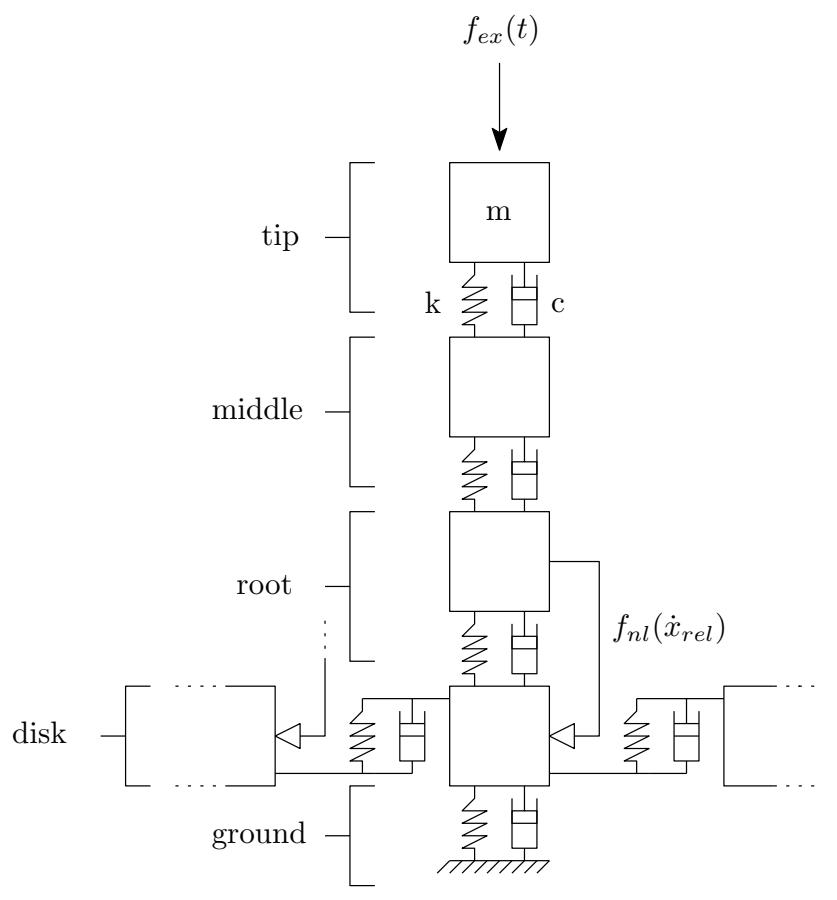

Figure 1: Fundamental sector of the lumped-parameter model with dry friction nonlinearity between the blade root and the disk.

Table 1: Numerical values of the structural parameters used to build the fundamental sector of the model.

\begin{tabular}{lccccc}
\hline$-($ unit $)$ & Tip & Middle & Root & Disk & Ground \\
\hline $\mathrm{m}\left(10^{-3} \cdot \mathrm{kg}\right)$ & 5 & 5 & 15 & 120 & - \\
$\mathrm{c}(\mathrm{N} \cdot \mathrm{s} / \mathrm{m})$ & 0.1 & 0.1 & 0.1 & 0.1 & 0.1 \\
$\mathrm{k}\left(10^{5} \cdot \mathrm{N} / \mathrm{m}\right)$ & 6 & 10 & 300 & 100 & 25 \\
\hline
\end{tabular}

of the traveling wave, and is theoretically related to the modes which can be excited, through the number of nodal diameters of their mode shapes. Since the model consists of six coupled sectors, it may not accurately account for a high number of nodal diameters. As a consequence, the excitation retained in this paper for the computation of the forced response is a traveling sine wave with only one nodal diameter, acting on the blade tips. The expression of the excitation acting on the k-th blade $f_{e x}^{k}(t)$ is given by Eqn. (15), with $F$ the amplitude of the excitation, $\Omega$ the excitation frequency, and $N_{b}$ the number of blades.

$$
f_{e x}^{k}(t)=F \cos \left(\Omega t+\frac{2 \pi k}{N_{b}}\right)
$$

The normalized time series of the excitation over a period $\mathrm{T}$ is given on Fig. (2a), on which is noticeable the propagation of the wave from the sixth to the first blade tip, resulting in a characteristic diagonal pattern. At a given time step on the $\mathrm{x}$-axis, the spatial shape of the excitation amplitude over the sectors on the $\mathrm{y}$-axis clearly exhibits one nodal diameter, as illustrated on Fig. (2b).

\subsection{Friction law}

To compute the nonlinear forces arising from a friction nonlinearity, several models are available. Coulomb's dry friction law is retained here for its simplicity, but others can be found in the literature, such as those of Dahl and Bouc-Wen. One simple way to implement Coulomb's friction law for one-dimensional models is to use a sign function taking the relative velocities of the DOFs in contact as arguments. In this paper, 
Table 2: Natural frequencies of the first family of bending modes of the tuned linear system for bonded and frictionless interfaces.

\begin{tabular}{lcccccc}
\hline$f_{i}(\mathrm{~Hz})$ & $f_{1}$ & $f_{2}$ & $f_{3}$ & $f_{4}$ & $f_{5}$ & $f_{6}$ \\
\hline Bonded & 654 & 1202 & 1202 & 1257 & 1257 & 1261 \\
Frictionless & 653 & 1189 & 1189 & 1244 & 1244 & 1248 \\
\hline
\end{tabular}

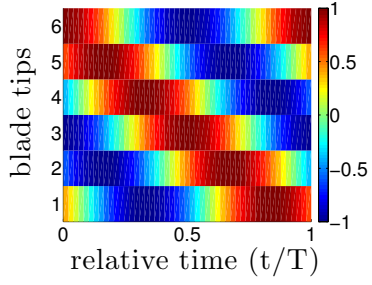

(a) Time series

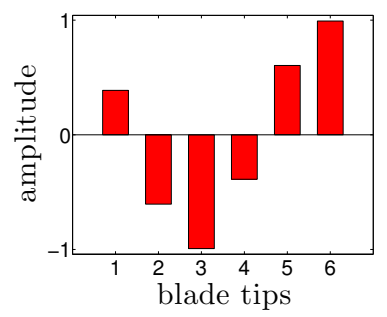

(b) Spatial shape at $\mathrm{t} / \mathrm{T}=0$

Figure 2: Traveling wave excitation used to compute the forced response

the sign function is approximated by a hyperbolic tangent, allowing for the analytical jacobian matrix of the algebraic system (5) to be readily obtained. In the time domain, the nonlinear forces acting on the frictional DOFs are thus given by Eqn. (16) and Eqn. (17).

$$
\begin{aligned}
\mathbf{f}_{n l}(\dot{\mathbf{x}}) & =\mu N \tanh \left(\frac{\dot{\mathbf{x}}_{r e l}}{\varepsilon}\right) \\
\dot{\mathbf{x}}_{r e l} & =\mathbf{P} \dot{\mathbf{x}}
\end{aligned}
$$

The scalars $\mu$ and $N$ are constant parameters, representing the friction coefficient and the normal load enforcing the contact, respectively. The friction being a function of the relative motion between the DOFs in contact, the vector of relative velocities $\dot{\mathbf{x}}_{r e l}$ is obtained from $\dot{\mathbf{x}}$ by means of a change-of-basis matrix $\mathbf{P}$, achieving the transformation from absolute to relative coordinates. The normal load is taken equal to the centrifugal load acting on a blade, that is approximately $1 \mathrm{kN}$, and a friction coefficient of 0.3 is kept throughout the study. The parameter $\varepsilon$ defines the degree of regularization, the smaller $\varepsilon$ the better the approximation of the sign function, as shown on Fig. (3), but the longer the convergence of the iterative solver. In order to account of the sticking state at low vibration levels, a relatively small value of $\varepsilon$ is required, otherwise the hyperbolic tangent function would amount to the first order to adding a viscous damping equal to $\mu N / \varepsilon$. On the other hand, at high vibration level, the value retained for $\varepsilon$ at low amplitude would make the convergence of the solver extremely hard, with yet no significant changes in the solution. This parameter is thus chosen so as to ensure a good compromise between computational cost and accuracy, varying from $10^{-5}$ at low amplitude to $10^{-3}$ at high amplitude.

\section{Results and discussion}

The results obtained in the study of the lumped-parameter model are presented and discussed in the following section. The nonlinear complex modes are computed by means of the methodology presented above, and the forced response is obtained with a standard multi-harmonic balance method i.e. by adding the traveling wave excitation of Eqn. (15) to Eqn. (1) for each blade tip, while dropping the exponential term in Eqn. (2). First, the convergence of the forced response as a function of the number of retained harmonics is addressed.

\subsection{Number of harmonics}

One of the most important parameter of the multi-harmonic balance method is the number $N_{h}$ of retained harmonics in the series expansion of the solution $\mathbf{x}(t)$, given in Eqn. (2). A good understanding of the nature of the nonlinearity can help to make some useful assumptions as to which harmonics to keep. For instance, 


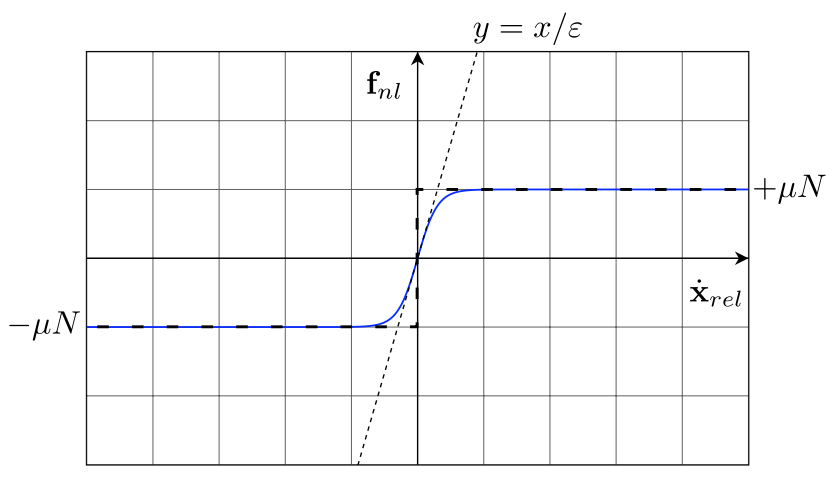

Figure 3: Regularized friction law governing the nonlinearity $(-)$.

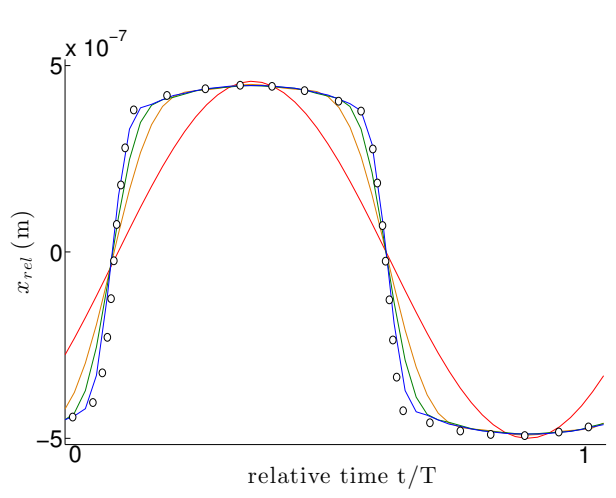

(a) Relative displacement

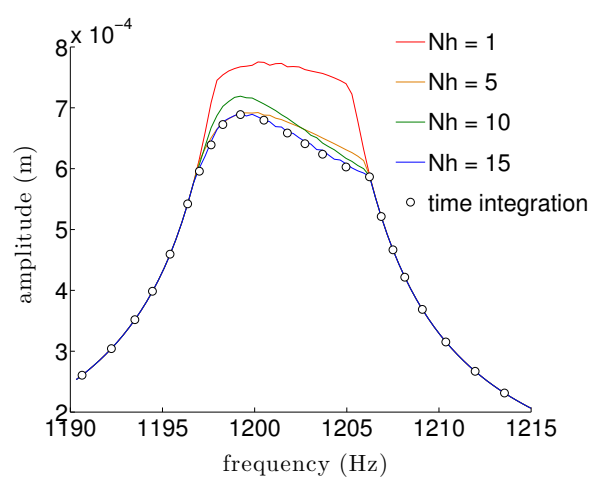

(b) Amplitude at the tip

Figure 4: Influence of the number of retained harmonics on the accuracy of the solution.

a cubic stiffness only generates odd harmonics, which allows to significantly reduce the size of the algebraic system by dropping all even harmonics [25]. For a dry friction nonlinearity, the key feature defining the quality of the solution is the accuracy with which the relative motion between the DOFs in contact can be approximated. In other words, a sufficient number of harmonics must be kept to account for the stick-slip transitions occurring at the interface when the nonlinearity is activated. To illustrate this, the relative motion between two frictional DOFs is plotted on Fig. (4a) for different values of $N_{h}$, at a fixed level of excitation $F=2$ newtons, strong enough for some slipping to occur. The relative displacement computed by HBM clearly converges toward the pattern yielded by a time integration, several harmonics being required to ensure the quality of the approximation. The accuracy with which this pattern is approximated directly affects the response in amplitude, as illustrated on Fig. (4b). With that in mind, fifteen harmonics are kept in this study, as a compromise between accuracy and computational efficiency.

\subsection{Tuned system}

The forced response and nonlinear complex modes are computed in the case of a tuned system, that is when no structural discrepancies break the cyclic symmetry of the model. It provides information as to how the friction impacts the dynamic of the perfectly periodic structure, and is used as a reference to interpret the dynamics of the mistuned system in both free and forced response.

Due to the nature of the excitation, a single resonance occurs in the surroundings of the first pair of modes of the underlying linear system i.e. the pair of modes with a deformed shape exhibiting one nodal diameter. The forced response of the system is given on Fig. (5) around the natural frequency of this pair of modes, for different excitation levels $F \in[0.1,5]$ newtons. The time series of the response at the blade tips is 


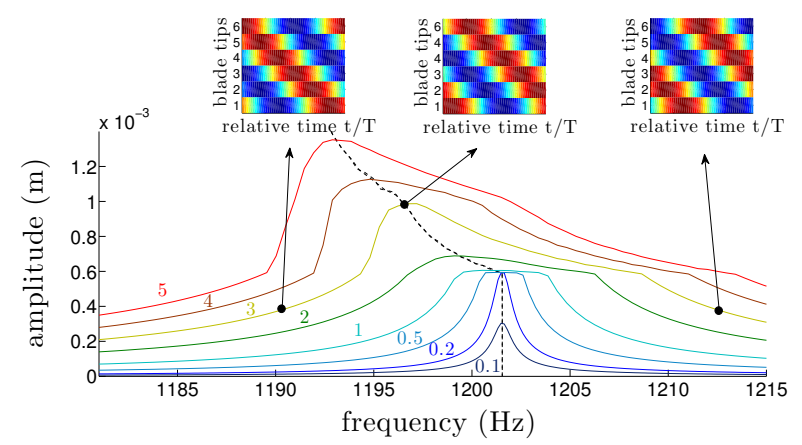

Figure 5: Forced response and time series of the tuned system for different excitation levels $F$.

provided at different points, and show that the response to the excitation is also a traveling wave propagating in the same direction. A phase lag between the excitation and the response can be seen on these time series, and varies from 0 to $\pi$ radians far before and far after the resonance respectively, as commonly observed in linear dynamics. The free response corresponding to the two nonlinear modes is plotted on the same graph with dashed lines. It can be noticed that the so-called backbone curves of the modes are superimposed and trace the locus of the maxima of the forced response, as already observed for non-cyclic systems [17, 22].

A further analysis of the nonlinear modes on Fig. (6) points out their energy-dependency, which among other things is embodied by the natural frequency being a function of the amplitude of vibration. For each mode, at low amplitude (in the vicinity of point A on Fig. (6)), the natural frequency is constant and equal to that of the underlying linear system with bonded DOFs at the interface. When the amplitude is high enough to activate the nonlinearity, i.e. to introduce some slipping between the DOFs in contact, the natural frequency gradually shifts toward a lower vertical asymptote, corresponding to the linear case with frictionless interface (in the vicinity of point B on Fig. (6)). That is why friction is commonly referred to as a softening nonlinearity.

In linear modal analysis of cyclic structures, it is known that the two orthogonal mode shapes corresponding to a double mode can be linearly combined to obtain two traveling sine waves propagating in opposite directions. As a consequence, the forced response to a traveling wave excitation in the vicinity of the natural frequency of a double mode is a traveling wave, linear combination of these orthogonal modes. The time series and mode shapes of the two nonlinear modes of interest here are given on Fig. (6), obtained by computing the displacement vector $\mathbf{x}$ from Eqn. (2). It shows that in that case, both modes are traveling waves propagating in opposite directions, for all amplitudes of vibration i.e. in the linear domain as well as in the nonlinear domain. This points out that even though the nonlinearity has a significant impact on the natural frequency, it does not affect the potentially propagative nature of the double mode of the underlying linear system. In other words, the two nonlinear complex modes originating from a pair of linear modes can still be combined to obtain traveling waves, even for a strong activation of the dry friction. Consequently, when subjected to a traveling wave excitation, the time series of the forced response at resonance in the nonlinear domain is still a traveling wave, as observed on Fig. (5) for $F=3$ newtons. This result should be remembered when analyzing the mistuned system in the next section.

Furthermore, even though at the blade tips scale the mode shapes do not seem to vary with the amplitude, they necessarily do since the frictional DOFs are in a stationary sticking state at low amplitude (i.e. in the vicinity of point A on Fig. (6)), and gradually shift toward a slipping state as the amplitude increases (i.e. in the vicinity of point B on Fig. (6)). Figure (7) shows a close-up on the interface and confirms this evolution of the mode shapes along the backbones at a smaller scale. As a consequence, the subspaces of the configuration space corresponding to these two free motions are curved, and both modes are therefore nonsimilar according to Rosenberg's terminology.

In the study of frictional systems, it is common to plot the compliance of the response for different 


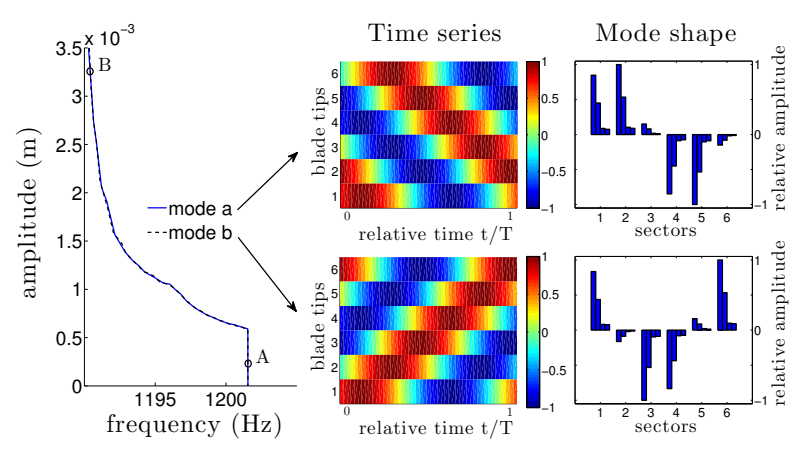

Figure 6: Backbone curves (superimposed), time series, and mode shapes at $\mathrm{t} / \mathrm{T}=0$ of the first pair of modes of the tuned system.
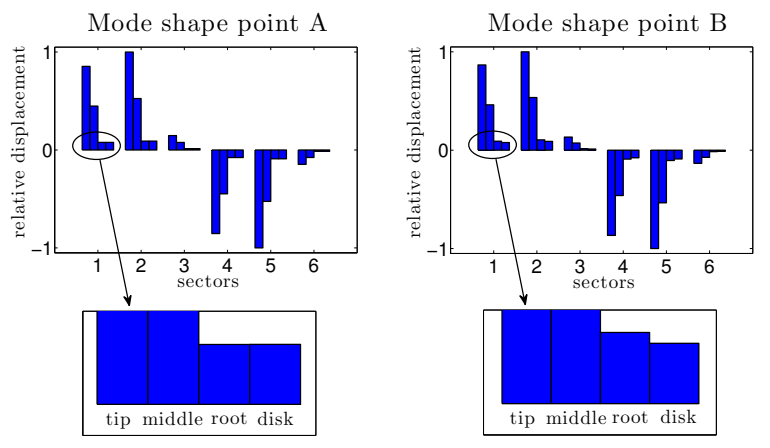

Figure 7: Close-up on the mode shape of mode "a" at $\mathrm{t} / \mathrm{T}=0$ for different amplitude levels.

activation levels of the nonlinearity, here embodied by the excitation amplitude $F \in[0.2,15]$ newtons, in order to exhibit the increase in damping together with the resonance shift induced by the friction, as on Fig. (8). The behavior obtained in this specific case is rather standard [2, 28], with an optimum in terms of damping for an excitation level $F$ of about 5 newtons noticeable on the graph. To plot this network of curves, several forced response computations are required, whereas the graph only yields an overview of the evolution of the damping. A useful by-product of the computation of nonlinear complex modes is the precise evolution of the modal damping $\beta$ as a function of the other variables $\omega$ and $\hat{\mathbf{x}}$. Figure (9) shows the evolution of this nonlinear damping as a function of the amplitude of vibration of the blades. At low amplitude (i.e. in the vicinity of point $\mathrm{A}$ ), the damping is constant and equal to that of the underlying linear system with bonded interfaces, since no slipping occurs. At high amplitude (i.e. in the vicinity of point B), $\beta$ slowly converges toward the value of the linear system with frictionless interfaces. Between these two limit cases, in the partial-slip area, a maximum in damping occurs, as commonly pointed out in the literature for non cyclic systems[22]. After a first local maximum, a new increase in $\beta$ is observed, which can be explained by a change in the relative motion at the interface. The transition from the first to the second peak corresponds indeed to the occurrence of two additional stuck phases in the relative displacement between two DOFs in contact, as illustrated on Figure (9).

The computation of nonlinear complex modes shows that an accurate optimization of the nonlinearity, with regard to vibration mitigation, is also possible for periodic structures, and that the modal damping of such systems may exhibit local extrema. In the next section, the results obtained for a mistuned system are presented and discussed.

\subsection{Mistuned system}

In this section, some structural mistuning is added to the model through a random pattern of discrepancies on the blades tip and middle stiffnesses. For a given stiffness, the mistuned value $k_{m}$ is derived from the tuned 


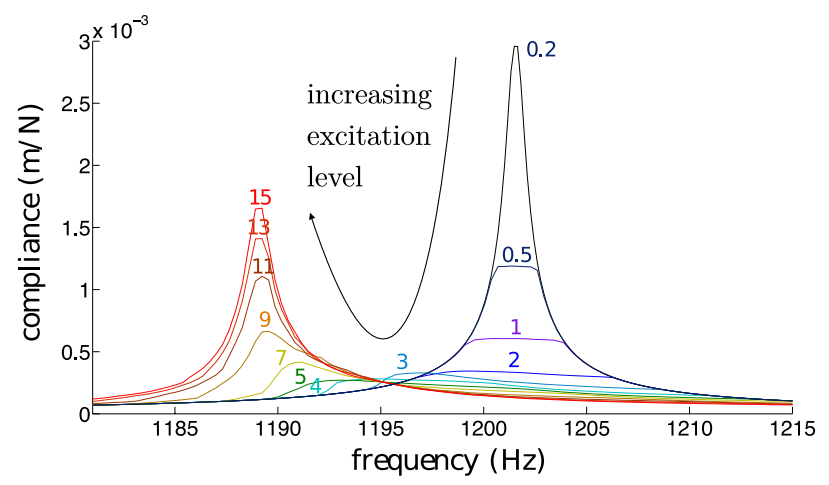

Figure 8: Compliance at the blade tips of the tuned system for different excitation levels $F$.

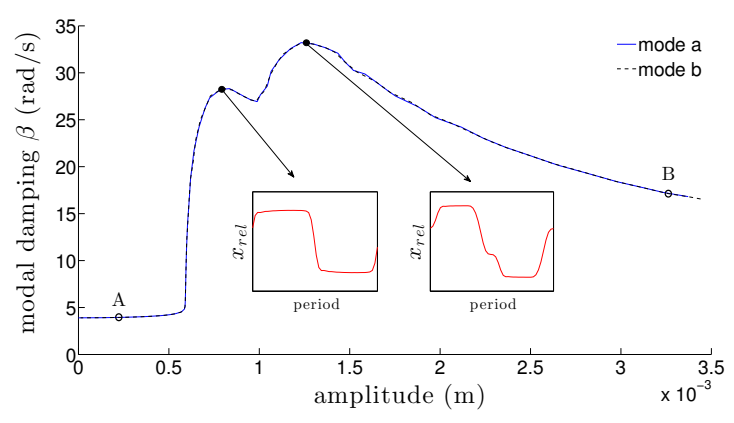

Figure 9: Modal damping curves (superimposed) of the first pair of modes of the tuned system as a function of the amplitude of vibration at the blade tips.

value $k_{t}$ by addition of a random perturbation $\xi$ taken in the continuous uniform distribution $\left[-10^{-3}, 10^{-3}\right]$. The perturbation is multiplied by a coefficient $d_{m}$ referred to as "mistuning level" and defining the mistuning magnitude. This coefficient is taken equal to 100 in this section, resulting in a $2 \%$ split of the first pair of natural frequencies of the tuned linear system, previously given in Tab. (2). The natural frequencies of the mistuned model are reported in Tab. (3).

$$
k_{m}=k_{t} \cdot\left(1+d_{m} \cdot \xi\right)
$$

For the same traveling wave excitation than in the study of the tuned system, the forced response no longer exhibits a single resonance, but several peaks of variable amplitudes throughout the frequency range. This can be explained by taking a look at the mode shapes of the mistuned system. As a consequence of the mistuning, each pair of modes of the underlying linear system is split into two modes with distinct natural frequencies, the time series of which are sheer stationary waves, which cannot be linearly combined so as to obtain traveling sine waves, as in the tuned case. Thus, the traveling nature of the excitation cannot be perfectly accounted for any longer by a double mode, and every mode brings a contribution to the response, which explains the multiplicity of the resonances.

Nevertheless, the greatest contribution to the response (i.e. the main peak) is observed in the surroundings of the two modes originating from the splitting of the former pair with one nodal diameter. In this frequency range, the forced response at one of the blade tips is given on Fig. (10) for different excitation levels $F \in[0.1,5]$. The time series of the response is provided at specific points, disclosing that far from resonance, the response can be split into a traveling wave (i.e. a diagonal pattern) and a stationary wave. As the response approaches a resonance, the traveling contribution gradually vanishes, and finally yields a single stationary wave when is reached the local maximum. This behavior of the time series is made for 
Table 3: Natural frequencies of the first family of bending modes of the mistuned linear system for bonded and frictionless interfaces.

\begin{tabular}{lcccccc}
\hline$f_{i}(\mathrm{~Hz})$ & $f_{1}$ & $f_{2}$ & $f_{3}$ & $f_{4}$ & $f_{5}$ & $f_{6}$ \\
\hline Bonded & 654 & 1176 & 1199 & 1237 & 1255 & 1285 \\
Frictionless & 653 & 1165 & 1187 & 1225 & 1242 & 1272 \\
\hline
\end{tabular}
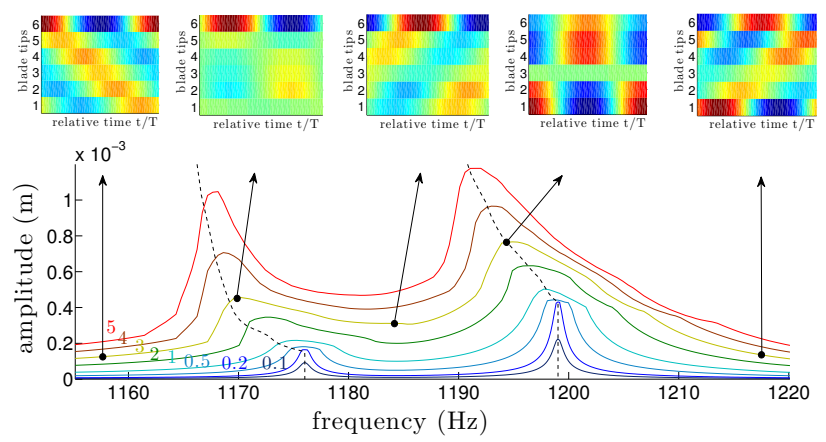

Figure 10: Forced response and time series of blade 2 of the mistuned system for different excitation levels $F$.

all excitation levels. The free response associated to the two modes is plotted in dashed-lines on the same graph. For a specific mode, the backbone traces with more or less accuracy the locus of the maxima, the slight difference being accounted for by the non-negligible contribution of the neighboring mode.

A focus on the nonlinear modes allows to better understand the time series of the forced response. On Fig. (11) is given the time series and mode shapes of the two modes of interest. It appears that both modes are indeed stationary waves, the time series of which closely match the time series of the forced response at each resonance arising from the corresponding mode. Thus, it can be inferred that the stationary component of the forced response arises from the closest mode, whereas the traveling part is a by-product of the excitation, built from other modal contributions. Due to the frequency split brought by the mistuning, the traveling wave excitation yields a sheer stationary response at resonance, in contrast to the tuned linear and nonlinear cases. It should be noticed that in that specific case, even though the mistuning impacts the traveling nature of the response at resonance, both mode shapes still feature one nodal diameter (one of which exhibiting a significant localization) and as a consequence generate the main peaks.

The compliance of the response is finally plotted on Fig. (12) for $F \in[0.2,13]$ newtons, showing that the behavior of both resonances follows the same standard pattern than the tuned system, though the level of activation of the nonlinearity yielding the optimum damping for one resonance may not yield an optimum damping for the second resonance. Should both resonances be critical for the structure, and a compromise would be needed. Finding a criterion to assess the quality of such a compromise is beyond the scope of this paper. However, the computation of the nonlinear complex modes could be of great interest to define this criterion and optimize the mitigation, through the evolution of the modal damping $\beta$ shown on Fig. (13). One could for instance apply weight functions to the modal damping of each mode, according to the relative importance of their respective resonances.

The forced responses presented so far are those monitored at a specific blade tip of the model. However, in the case of a mistuned system, each blade exhibits a peculiar response which can significantly differ from one another. On Fig. (14) is given the forced response of another blade tip for different levels of excitation $F \in[0.1,5]$ newtons. For that blade, the first resonance exhibits a less standard behavior, with one peak gradually splitting into two distinct peaks, and finally resulting in a single peak as the excitation keeps increasing. The computation of the nonlinear modes and a close look at the time series of both the forced 


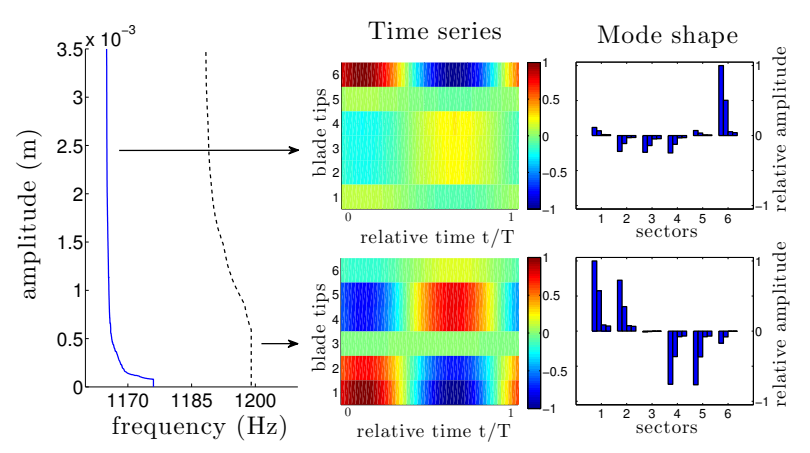

Figure 11: Backbone curves, time series, and mode shapes at $\mathrm{t} / \mathrm{T}=0$ of the first pair of modes split by the mistuning.

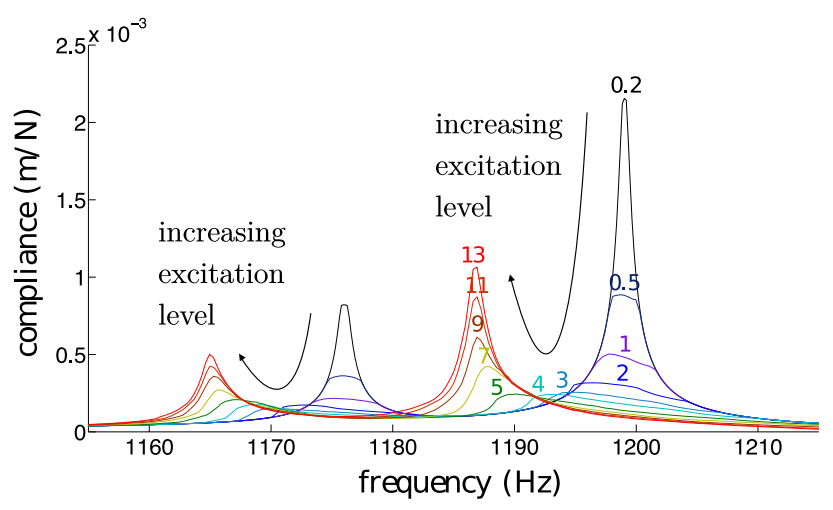

Figure 12: Compliance at the tip of blade 2 of the mistuned system for different excitation levels $F$.

response and the backbones permit to understand that the two peaks actually result from a single resonance, even though the mechanisms at the origin of such a behavior have not yet been tackled.

\subsection{Qualitative analysis of the mistuning magnitude}

A great deal of statistical studies have been carried out in linear dynamics, and revealed that the mistuning is accountable for an amplification of the global response of the structure, with a variable sensitivity of the amplification to the degree of mistuning [12]. However, similar investigations for nonlinear systems have seldom been undertaken due to significant computational costs, but could yet help to improve the robustness of the design. By means of the model studied in this paper, the amplification resulting from the mistuning is determined for the random mistuning pattern used in the previous section, the magnitude of which is gradually increased through the coefficient $d_{m}$ of Eqn. (18), referred to as "mistuning level" hereafter. The nonlinearity is taken into account for both tuned and mistuned systems, and the excitation is kept at a constant level $F=2$ newtons, chosen so as to bring the system in the nonlinear domain (see for instance Fig. (10) or Fig. (12)). The analysis is also performed for a second random mistuning pattern in order to catch a glimpse of a potential common behavior. The results are plotted on Fig. (15), and show that for this specific case, the amplification increases more abruptly at low mistuning levels for both patterns, one of which exhibiting what could be interpreted as an overshoot. Should this observation be made for all kind of mistuning patterns by means of further statistical investigations, and the robustness of the design could be improved by willingly avoiding the range of high sensitivity. In linear dynamics of bladed disks, this has led to what is usually referred to as intentional mistuning. However, at the present time, too few data are available to extend this notion to nonlinear systems.

Another matter of interest when it comes to combining mistuning and dry friction is the influence of the mistuning on the vibration mitigation arising from the nonlinearity. For a fixed mistuning pattern, the 


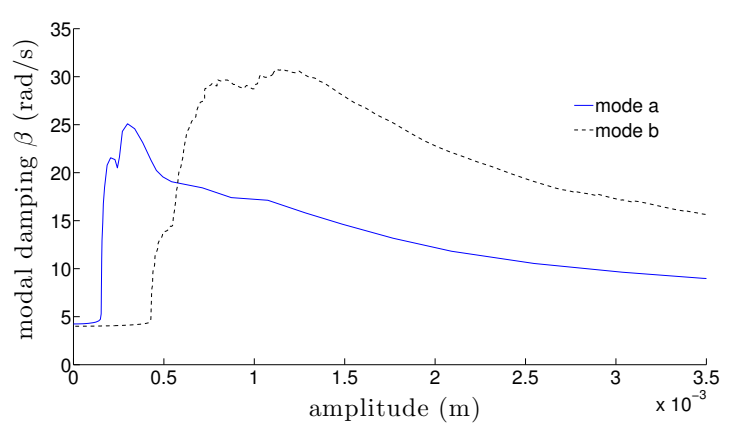

Figure 13: Modal damping curves of the first pair of modes split by the mistuning as a function of the amplitude of vibration of blade 2 .

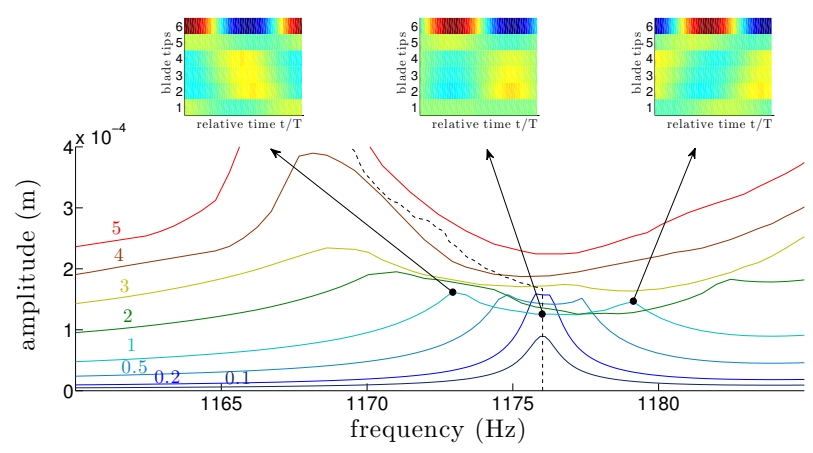

Figure 14: Response at the tip of another blade of the mistuned system for different excitation levels $F$.

damping resulting from the activation of the nonlinearity may indeed vary with the mistuning magnitude. The maximum amplitude of the mistuned nonlinear system is thus compared to the maximum amplitude of the underlying mistuned linear system with bonded interface for different mistuning levels. The results on Fig. (16) show that for these two specific mistuning patterns, the optimum damping arising from the friction is not obtained for a tuned system, but for a slightly mistuned structure. Should this result be extended to all mistuned structures subjected to dry friction by further statistical investigations, and the design of bladed disks could take great advantage in finding an optimum mistuning magnitude to mitigate vibrations.

\section{Conclusion}

The phenomenology of periodic structures subjected to dry friction nonlinearities has been investigated by means of a lumped-parameter model representative of turbomachinery bladed disks. The computation of the nonlinear complex modes of the system has proved very helpful to interpret the forced response to a traveling wave excitation, especially when some structural mistuning breaks the cyclic symmetry of the assembly. Nonlinear complex modes are also of great interest in seeking an optimal vibration mitigation, by providing information as to how the friction impacts the modal damping of such nonlinear systems.

The influence of the mistuning magnitude on the amplification generated by the mistuning and on the mitigation arising from the friction has also been addressed. It appears that for such nonlinear systems, the robustness of the design may be improved by adding some intentional mistuning, as already observed for linear models. An optimum mistuning magnitude has also been detected in terms of vibration mitigation, for two random mistuning patterns.

Providing industrials with new tools, allowing for the determination of the forced response of large-scale, mistuned, and nonlinear systems, at relatively low computational cost, could significantly help to improve the 


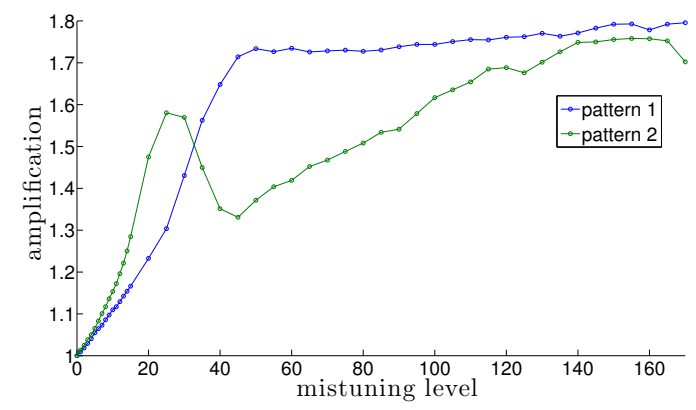

Figure 15: Amplification factor between the mistuned and tuned nonlinear systems for $F=2$ newtons.

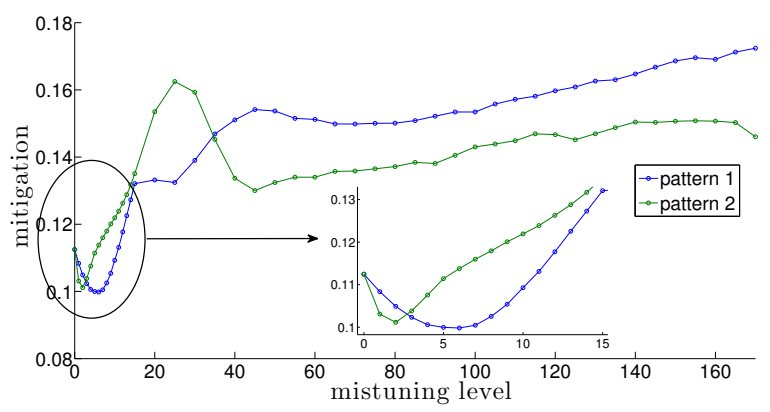

Figure 16: Mitigation factor between the nonlinear and underlying linear mistuned systems for $F=2$ newtons.

design of bladed disks. However, the study of the combined effects of structural mistuning and dry friction is a rich and complex topic, and further qualitative and quantitative investigations by means of more realistic models are still necessary. Conducting statistical studies on random mistuning patterns could also help to extend the useful notion of intentional mistuning to nonlinear structures.

\section{Acknowledgment}

The authors are grateful to Turbomeca for providing the financial support for this project, and for giving permission to publish this work.

\section{References}

[1] E. Petrov, D. Ewins, Effects of damping and varying contact area at bladed-disk joints in forced responses analysis of bladed disk assemblies, J Turbomach 128 (2006) 403 - 410. doi:10.1115/1.2181998.

[2] D. Laxalde, F. Thouverez, J.-J. Sinou, J.-P. Lombard, Qualitative analysis of forced response of blisks with friction ring dampers, European Journal of Mechanics - A/Solids 26 (4) (2007) 676 - 687. doi: $10.1016 / j$. euromechsol.2006.10.002.

[3] L. Salles, L. Blanc, F. Thouverez, A. Gouskov, Dynamic analysis of fretting-wear in friction contact interfaces, International Journal of Solids and Structures 48 (10) (2011) 1513 - 1524. doi:10.1016/j. ijsolstr.2011.01.035.

[4] S. Nacivet, C. Pierre, F. Thouverez, L. Jezequel, A dynamic lagrangian frequency-time method for the vibration of dry-friction-damped systems, Journal of Sound and Vibration 265 (1) (2003) 201 - 219. doi:10.1016/S0022-460X (02) 01447-5. 
[5] D. Charleux, C. Gibert, F. Thouverez, J. Dupeux, Numerical and experimental study of friction damping in blade attachments of rotating bladed disks, International Journal of Rotating Machinery 2006 (2006) 1 - 13. doi:10.1155/IJRM/2006/71302.

[6] T. Berruti, C. Firrone, M. Gola, A test rig for noncontact traveling wave excitation of a bladed disk with underplatform dampers, J Eng Gas Turb Pwr 133 (2011) 032502-1-8. doi:10.1115/1.4002100.

[7] S. Zucca, D. D. Maio, D. Ewins, Measuring the performance of underplatform dampers for turbine blades by rotating laser doppler vibrometer, Mechanical Systems and Signal Processing 32 (2012) $269-281$.

[8] C. Schwingshackl, E. Petrov, D. Ewins, Measured and estimated friction interface parameters in a nonlinear dynamic analysis, Mechanical Systems and Signal Processing 28 (0) (2012) 574 - 584. doi: $10.1016 / j \cdot y m s s p .2011 .10 .005$.

[9] C. Schwingshackl, C. Joannin, L. Pesaresi, J. Green, N. Hoffmann, Test method development for nonlinear damping extraction of dovetail joints, Dynamics of Coupled Structures 1 (2014) 229 - 237. doi:10.1007/978-3-319-04501-6_21.

[10] C. Pierre, E. Dowell, Localization of vibrations by structural irregularity, Journal of Sound and Vibration 114 (3) (1987) 549-564. doi:10.1016/S0022-460X (87)80023-8.

[11] M. P. Castanier, C. Pierre, Using intentional mistuning in the design of turbomachinery rotors, AIAA Journal 40 (10) (2002) 2077-2086. doi:10.2514/2.1542.

[12] M. Mbaye, C. Soize, J.-P. Ousty, E. Capiez-Lernout, Robust analysis of design in vibration of turbomachines, J Turbomach 135 (2013) 021008-1-8. doi:10.1115/1.4007442.

[13] F. Pichot, D. Laxalde, J.-J. Sinou, F. Thouverez, J.-P. Lombard, Mistuning identification for industrial blisks based on the best achievable eigenvector, Computers \& Structures 84 (29-30) (2006) 2033 - 2049. doi:10.1016/j.compstruc.2006.08.022.

[14] P. Vargiu, C. Firrone, S. Zucca, M. Gola, A reduced order model based on sector mistuning for the dynamic analysis of mistuned bladed disks, International Journal of Mechanical Sciences 53 (8) (2011) 639 - 646. doi:10.1016/j.ijmecsci.2011.05.010.

[15] D. Ewins, Modal Testing : Theory Practice and Application, 2nd Edition, Research Study Press LTD, Baldock, England, 2000.

[16] R. Rosenberg, The normal modes of nonlinear n-degrees-of-freedom systems, Journal of Applied Mechanics 30 (1962) $595-611$. doi:10.1115/1.3636501.

[17] G. Kerschen, M. Peeters, J. Golinval, A. Vakakis, Nonlinear normal modes, part i: A useful framework for the structural dynamicist, Mechanical Systems and Signal Processing 23 (1) (2009) 170 - 194. doi: $10.1016 / j \cdot y m s s p .2008 .04 .002$.

[18] S. Shaw, C. Pierre, Normal modes for non-linear vibratory systems, Journal of Sound and Vibration 164 (1) (1993) 85 - 124. doi:10.1006/jsvi.1993.1198.

[19] A. F. Vakakis, Dynamics of a nonlinear periodic structure with cyclic symmetry, Acta Mechanica 95 (1-4) (1992) 197-226. doi:10.1007/BF01170813.

[20] A. F. Vakakis, C. Cetinkaya, Mode localization in a class of multidegree-of-freedom nonlinear systems with cyclic symmetry, SIAM J. Appl. Math. 53 (1) (1993) 265-282. doi:10.1137/0153016.

[21] A. Vakakis, T. Nayfeh, M. King, A multiple-scales analysis of nonlinear, localized modes in a cyclic periodic system, Journal of Applied Mechanics 60 (2) (1993) 388-397. doi:10.1115/1.2900806.

[22] D. Laxalde, F. Thouverez, Complex non-linear modal analysis for mechanical systems: Application to turbomachinery bladings with friction interfaces, Journal of Sound and Vibration 322 (4-5) (2009) 1009 - 1025. doi:10.1016/j.jsv.2008.11.044. 
[23] M. Krack, L. P. von Scheidt, J. Wallaschek, A method for nonlinear modal analysis and synthesis: Application to harmonically forced and self-excited mechanical systems, Journal of Sound and Vibration 332 (25) (2013) 6798 - 6814. doi:10.1016/j.jsv.2013.08.009.

[24] S.-T. Wei, C. Pierre, Effects of dry friction damping on the occurrence of localized forced vibrations in nearly cyclic structures, Journal of Sound and Vibration 129 (3) (1989) 397 - 416. doi:10.1016/ 0022-460X (89) 90432-X.

[25] A. Grolet, F. Thouverez, Free and forced vibration analysis of a nonlinear system with cyclic symmetry: Application to a simplified model, Journal of Sound and Vibration 331 (12) (2012) 2911 - 2928. doi: $10.1016 / j \cdot j$ sv.2012.02.008.

[26] T. M. Cameron, J. H. Griffin, An alternating frequency/time domain method for calculating the steadystate response of nonlinear dynamic systems, Journal of Applied Mechanics 56 (1) (1989) 149-154. doi:10.1115/1.3176036.

[27] A. Nayfeh, B. Balachandran, Applied nonlinear dynamics: analytical, computational and experimental methods, Wiley-Interscience, 1995.

[28] E. Petrov, D. Ewins, Analytical formulation of friction interface elements for analysis of nonlinear multiharmonic vibrations of bladed disks, J Turbomach 125 (2003) 364 - 371. doi:10.1115/1.1539868. 